\title{
Glutamate decarboxylase (GAD65) and tyrosine phosphatase-like protein (IA-2) autoantibodies index in a regional population is related to glucose intolerance and body mass index
}

\author{
O. Rolandsson ${ }^{1}$, E. Hägg ${ }^{2}$, C. Hampe ${ }^{3}$, E. P. Sullivan Jr. ${ }^{3}$, M. Nilsson ${ }^{4}$, G. Jansson ${ }^{5}$, G. Hallmans ${ }^{6}$, Å. Lernmark ${ }^{3}$ \\ ${ }^{1}$ Department of Family Medicine, Umeå University, Sweden \\ ${ }^{2}$ Department of Medicine, Umeå University, Sweden \\ ${ }^{3}$ Department of Medicine, University of Washington, Seattle, Washington, USA \\ ${ }^{4}$ Department of Epidemiology, Umeå University, Sweden \\ ${ }^{5}$ Stenbergska Primary Health Care Centre, Lycksele, Sweden \\ ${ }^{6}$ Department of Nutritional Research, Umeå University, Sweden
}

\begin{abstract}
Aims/hypothesis. Our aims were to investigate the concentrations and prevalence of autoantibodies against the $\mathrm{Mr} 65.000$ isoform of glutamate decarboxylase (GAD65) and the tyrosine phosphatase-like protein (IA-2) in adults and to test the hypothesis that GAD65 and IA-2 autoantibodies in a regional population are related to abnormal oral glucose tolerance.

Methods. We analysed serum from 2157 Swedish subjects aged either $30,40,50$ or 60 years old who, in 1988-1992, participated in the Västerbotten County Health Project and were subjected to the World Health Organisation (WHO) standard oral glucose tolerance test at entry into the study.

Results. We found 23 of 2157 (1.1\%) and 17 of 2152 $(0.8 \%)$ subjects exceeded the 99th centile of GAD65 autoantibody index and IA-2 autoantibody index, respectively. In 18 subjects with diabetic oral glucose tolerance test, GAD65 autoantibody concentrations were higher than in those with normal oral
\end{abstract}

glucose tolerance test $(p=0.02)$. Subjects with IGT $(n=416)$ and diabetes $(n=18)$, i. e. abnormal OGTT $(n=434)$, had a higher IA-2Ab index compared with those with normal OGTT $(p=0.008)$. A stepwise multiple logistic regression test showed that the odds ratios for subjects in the highest BMI group to exceed the 95 th or 99th GAD65 autoantibody centile were 3.6 (CI 1.4-8.9) and 17.6 (CI 2.6-121.6), respectively. Conclusion/interpretation. GAD65 and IA-2 autoantibodies, are associated with impaired or diabetic glucose tolerance in an adult regional population. This observation together with the association between GAD65 autoantibody concentrations and body mass index indicate a possible relation between islet autoimmunity and beta-cell function abnormalities with obesity and insulin resistance. [Diabetologia (1999) 42: 555-559]

Keywords Autoimmunity, beta-cell function, BMI, epidemiology, sex, glutamate decarboxylase, IA-2, Type I and Type II diabetes, OGTT.
The clinical diagnosis of diabetes mellitus is based on specific criteria of blood glucose concentrations [1]. After diagnosis of the disease, clinical criteria are used to classify diabetes into Type I (insulin-dependent) or Type II (non-insulin-dependent) diabetes

Received: 2 July 1998 and in final revised form: 4 December 1998

Corresponding author: O. Rolandsson, Department of Family Medicine, NUS By 9A, 90185 Umeå, Sweden

Abbreviations: GAD65Ab, Autoantibodies to the $65 \mathrm{kD}$ glutamate decarboxylase isoform; IA-2Ab, autoantibodies to the tyrosine phosphatase-like protein, IA-2. mellitus. The classification of diabetes is mostly unambiguous in people younger than 20 years of age and in older patients who predominately have Type II diabetes. It is widely recognised, however, that Type I diabetes can develop at any age which complicates classification in the older patient. Type I diabetes in children and young adults is strongly associated with the presence of autoantibodies against the Mr 65 $\mathrm{kD}$ isoform of glutamate decarboxylase (GAD65) [2] and IA-2 (ICA512), a tyrosine phosphatase-like transmembrane protein $[3,4]$. The diagnostic sensitivity of GAD65Ab has been reported to be about $70-80 \%$ and the diagnostic specificity $99 \%$ in such patients [5]. Furthermore, in newly diagnosed Type 
II diabetic patients it has been found that $7-10 \%$ have GAD65Ab and that these antibodies predict the requirement for insulin treatment [6]. It has therefore been suggested that these patients represent a slow onset Type I diabetes or latent autoimmune diabetes in the adult (LADA) [7]. Many earlier studies have also reported that there is a relation between secondary failure to oral hypoglycaemic agents and islet cell antibodies (ICA) among Type II diabetic patients irrespective of the duration of the disease $[8,9]$. Taken together, these data suggest that Type I diabetes immune markers are present in some adult diabetic patients who fulfil the classification criteria for Type II diabetes.

Since only $10-20 \%$ of new onset Type I diabetic patients have a first degree relative with the disease and in order to understand the possible development of Type I diabetes in adults, it is important to determine the prevalence of GAD65Ab and IA-2Ab as the major islet autoimmune markers in the general population. We also wanted to test the hypothesis that the Type I diabetes markers, GAD65Ab and IA-2Ab, are associated with pathological OGTT as a marker for abnormal glucose metabolism. The standardised oral glucose tolerance test (OGTT) is recommended by the World Health Organisation (WHO) and is often used in screening healthy adults for diabetes.

In this study we determined GAD65Ab and IA$2 \mathrm{Ab}$ in a well defined group of subjects and related autoantibody frequency and concentrations to sex, age, body mass index (BMI) and OGTT. We have taken advantage of the standardised, highly sensitive and specific test for GAD65Ab and IA-2Ab using in vitro transcribed and translated human recombinant GAD65 and IA-2 $[10,11]$ and a population-based investigation of 2157 subjects being either $30,40,50$ or 60 years of age.

\section{Subjects and methods}

Subjects in the Västerbotten County Health Project. The subjects studied were invited on their 30th, 40th, 50th or 60th birthday and were from the community of Lycksele in the county of Västerbotten. In 1988-1992, all inhabitants who were of these ages were invited without exclusion criteria to participate in a health survey at Stenbergska Primary Health Care Centre, Lycksele. A total of 2314 [men/women (M/W) $1167 / 1147]$ subjects representing $74 \%$ of the eligible $(n=3128)$ population participated in the health survey. In the survey, 21 subjects were previously known diabetic patients, 35 had unknown fasting time and 101 did not complete the OGTT. Therefore, 157 (M/W 72/85) were excluded from our study, thereby including $69 \%$ of the eligible population. The distribution of men and women in the age groups is shown in Table 1 . The subjects visited the primary health care centre in the morning after fasting for 4-8 h. The height and weight of the subjects were measured to calculate their body mass index $\left(\mathrm{BMI}=\mathrm{kg} / \mathrm{m}^{2}\right)$. Serum was prepared and stored at $-80^{\circ} \mathrm{C}$ for
Table 1. Distribution of subjects and BMI by age and sex in the study group $(n=2157)$

\begin{tabular}{|c|c|c|}
\hline Sex and age & \multirow{2}{*}{$\frac{n}{290}$} & \multirow{2}{*}{$\begin{array}{l}\text { BMI, median (min-max) } \\
24(15-37)\end{array}$} \\
\hline Men & & \\
\hline 4 & 332 & $25(19-35)$ \\
\hline 5 & 254 & $26(19-39)$ \\
\hline 6 & 219 & $26(18-35)$ \\
\hline Men total & 1095 & $25(15-39)$ \\
\hline \multirow[t]{4}{*}{ Women } & 239 & $23(17-40)$ \\
\hline & 295 & $24(16-40)$ \\
\hline & 289 & $25(17-44)$ \\
\hline & 239 & $26(18-41)$ \\
\hline Women total & 1062 & $25(16-44)$ \\
\hline
\end{tabular}

up to 8 years before the assay of GAD65Ab and IA-2Ab. The subjects gave their written consent to the study and the study was approved by the ethics committee of the Medical Faculty, Umeå University, Sweden.

Oral glucose tolerance test. Capillary fasting plasma glucose was measured after fasting. Subjects with a fasting plasma glucose less than $7.8 \mathrm{mmol} / \mathrm{l}$ were offered an OGTT. Those with fasting plasma glucose $7.8 \mathrm{mmol} / \mathrm{l}$ or more $(n=6)$ were considered to have overt diabetes. They were offered a follow-up with a new analysis of their fasting plasma glucose to establish the diagnosis. They are included in the results under the category "Diabetes". Using the 1985 WHO recommendations for OGTT, the subjects were divided into groups of normal glucose tolerance (fasting plasma and 2-h plasma glucose $<7.8 \mathrm{mmol} / \mathrm{l}$ ), impaired glucose tolerance fasting plasma glucose $<7.8 \mathrm{mmol} / \mathrm{l}$ and 2-h plasma glucose $\geq 7.8$ to $<12.2$ $\mathrm{mmol} / \mathrm{l}$ ) and diabetes (fasting plasma glucose $\geq 7.8 \mathrm{mmol}$ or fasting plasma glucose $<7.8 \mathrm{mmol} / \mathrm{l}$ and 2 -h plasma glucose $\geq 12.2 \mathrm{mmol} / \mathrm{l}$ ).

Analysis of serum lipids. Venous blood samples were drawn from the subjects at the health care centre and were analysed, expressed in $\mathrm{mmol} / \mathrm{l}$ for total cholesterol $(\mathrm{n}=2138, \mathrm{M} / \mathrm{W}$ $1083 / 1055)$, HDL-cholesterol $(n=1501, \mathrm{M} / \mathrm{W} 767 / 734)$ and triglycerides $(n=2135, \mathrm{M} / \mathrm{W} 1080 / 1055)$.

Analysis of $G A D 65 A b$. The serum samples of 2157 subjects were analysed for GAD65Ab in a radioligand-binding assay described in detail elsewhere [10]. In short, human recombinant GAD65 was produced by in vitro transcription/translation and labelled with 35S-methionine [12]. Reduction in the background radioactivity was achieved by filtration of the GAD65 buffer mixture through a 1.2 micron RA $25 \mathrm{~mm}$ filter (Millipore, Bedford Mass., USA). The labelled GAD65 was incubated with serum in duplicate determinations. Protein ASepharose (Zymed, San Fransisco, Calif., USA) was used to separate bound antibody from free ${ }^{35} \mathrm{~S}-\mathrm{GAD} 65$. After repeated washings in Millipore Multiscreen-DV 1.25 micron Filtration Plates, the Protein A-Sepharose was transferred to vials and the radioactivity was counted in a liquid scintillation counter. Concentrations of GAD65Ab were expressed as a $\mathrm{GAD} 65 \mathrm{Ab}$ index to correct for inter-assay variation according to the following formula: GAD65Ab index $=(\mathrm{cpm}$ of the unknown sample - average cpm of two negative standards)/ (cpm of the positive standard - average of two negative standards). The positive and negative controls and two separate quality controls were run at the beginning and end of each 96well filterplate. The intra-assay coefficient of variation for du- 
plicate determinations was $8.2 \%$. Our laboratory participated in the 1996 GAD65Ab proficiency test in the auspices of the Immunology of Diabetes Society with $100 \%$ sensitivity and $100 \%$ specificity.

Analysis of $I A-2 A b$. The serum samples of 2151 subjects (6 sera missing) were analysed for IA-2Ab in a radioligand-binding assay identical to the GAD65Ab assay but using ${ }^{35} \mathrm{~S}$-labelled IA-2 as a tracer. The tracer was produced from the plasmid pICA512bdc kindly donated by Dr G. Eisenbarth.

Displacement of GAD65Ab. To evaluate the specificity of the autoantibody analysis, 103 subjects with a GAD65Ab index exceeding the 95th centile were selected along with 386 randomly selected subjects, matched for age and sex. The matching was planned to be $1: 4$, but in 26 subjects it was $1: 3$ due to lack of serum. In the competitive GAD65Ab analysis, 150 nmol/1 unlabelled recombinant human GAD65 (rhGAD65) (BioSyn, Stockholm, Sweden) was used to block the binding of ${ }^{35} \mathrm{~S}-\mathrm{GAD} 65$ to GAD65Ab. Unlabelled $150 \mathrm{nmol} / \mathrm{l}$ proinsulin was used as control.

Statistical evaluation. Results are presented as median, percentiles, mean, mean rank, standard deviation (SD), values of different test-statistics and $p$-values. A $p$-value less than 0.05 is considered to be significant and the null-hypothesis is rejected, unless stated otherwise. Tests used were Mann-Whitney U test, Students paired $t$ test, Kolmogorovs test for normality, Spearmans correlation test and stepwise multivariate logistic regression.

\section{Results}

Age, sex, OGTT and BMI. We did OGTTs in 2157 subjects (M/W 1095/1062). When the subjects were grouped according to WHO recommendations for OGTT we found that 1723 were normal, 416 had IGT and 18 diabetes (Table 2). There were more men $(n=951)$ than women $(n=772)$ with normal OGTT $(p<0.001$, binomial test) and more women $(n=282)$ than men $(n=134)$ with IGT $(p<0.001$, binomial test). No difference in sex prevalence was found in the diabetes group. Concentrations of fasting plasma glucose were not correlated to any of the autoantibodies. Distribution of BMI in the age groups is shown in Table 1.

Serum lipids. None of the serum lipids were correlated to the GAD65Ab or the IA-2Ab concentrations (Spearman's correlation test).

$G A D 65 A b$. The distribution of GAD65Ab-index in the study group was not normally distributed (Kolmogorovs $\mathrm{D}$ statistic, $p<0.001)$. The median GA$\mathrm{D} 65 \mathrm{Ab}$ index in the study group was $0.0(\mathrm{~min}-0.21$, max 1.38) with no significant differences between sex or age groups. We found 23 subjects $(1.1 \%)$ exceeded the 99th centile of GAD65Ab index defined as below. The GAD65Ab index in the different OGTT groups is shown in Table 2. Subjects in the diabetes group $(n=18)$ had higher GAD65Ab indexes than those
Table 2. Distribution of sex and results of GAD65Ab and IA$2 \mathrm{Ab}$ analyses in relation to OGTT in 2157 subjects aged 30-60 years

\begin{tabular}{lccc}
\hline Sex and autoantibody index & OGTT & & \\
\cline { 2 - 4 } & Normal & IGT & Diabetes \\
\hline Men & 951 & 134 & 10 \\
Women & 772 & 282 & 8 \\
Total & 1723 & 416 & 18 \\
GAD65Ab index & & & \\
Exceeding the 99th centile $(n)$ & 16 & 7 & 0 \\
IA-2Ab index & & & \\
Exceeding the $99^{\text {th }}$ centile $(n)$ & 12 & 5 & 0 \\
\hline
\end{tabular}

with normal OGTT ( $n=1723, p=0.020)$. Using stepwise multiple logistic regression showed that the odds ratios for subjects in the highest BMI group to exceed the 95th or the 99th GAD65Ab centile were 3.6 (C.I. 1.4-8.9) and 17.6 (C. I. 2.6-121.6), respectively.

GAD65Ab in subjects with normal OGTT. We calculated the GAD65Ab index upper limits of the subjects with a normal OGTT for the 95th centile; cutoff level 0.08 exceeded by 73 subjects and the 99th centile: cut-off level 0.17 exceeded by 16 subjects. There was no correlation between GAD65Ab index and age in either women or men. When calculated in subjects ( $n=1701,22$ missing) with normal OGTT BMI was not normally distributed. In support of the logistic regression analysis, comparing subjects above and below the 99th centile of GAD65Ab index showed that the BMI was higher in 40-year-old men above $(n=11$; median BMI 27, min 26, max 35) than below $(n=281$, median BMI 25, min 20, $\max 35$; $p<0.001)$ and 60 -year-old women above $(n=6$; median BMI 30, min 26, max 35) compared with subjects matched to them for sex and age below $(n=148$, median BMI 25, min 18, $\max 41 ; p=0.016)$. In subjects exceeding the 99th centile of the GAD65Ab index also showed that 50-year-old women above $(n=5$, median BMI 29, min 23, max 33) had higher BMI than subjects below the 99th centile $(n=197$, median BMI 25, $\min 17, \max 37, p=0.05$ ).

GAD65Ab in subjects with abnormal OGTT. The number of subjects exceeding the 99th centile of GA$\mathrm{D} 65 \mathrm{Ab}$ index with IGT $(n=416, \mathrm{M} / \mathrm{W} 134 / 282)$ or diabetes $(n=18, \mathrm{M} / \mathrm{W} 10 / 8)$, i.e. abnormal OGTT $(n=434)$ is shown in Table 2 . There was no correlation between GAD65Ab index, age or sex. Comparing subjects above and below the 95th and the 99th centile of GAD65Ab index showed that the BMI was higher in subjects above $(n=21$; median BMI 27, min 21, max 40 and $n=7$; median BMI 29, min 25 , max 40 respectively) than below $(n=399$, median BMI 25, min 17 and max 44; $p=0.028$ and $n=413$; median BMI 25, min 17 and max 44, respectively, $p=0.028$ and 0.032 respectively). 
$I A-2 A b$. The distribution of IA-2Ab index in the study group was not normally distributed (Kolmogorovs $\mathrm{D}$ statistic, $p<0.001)$. The median IA-2Ab index was 0.0 ( $\min -0.02$, max 0.77 ) with no significant differences between sex or age groups. No relation was shown between IA-2Ab index and BMI or serum lipids. We found 17 subjects $(0.8 \%)$ exceeded the 99 th centile of IA-2Ab index defined as below. The IA$2 \mathrm{Ab}$ index in the different OGTT groups is shown in Table 2.

$I A-2 A b$ in subjects with normal OGTT. We calculated the different IA-2Ab index upper limits in subjects with a normal OGTT $(n=1719)$ : cut-off level for the 95th centile was 0.01 exceed by 40 subjects and the 99th centile: cut-off level 0.03 exceeded by 12 subjects. There was no correlation between IA-2Ab index and age in either women or men. We found IA$2 \mathrm{Ab}$ index was not correlated to BMI.

IA-2Ab in subjects with abnormal OGTT. Calculation of the different IA-2Ab index upper limits showed for the 95th centile a cut-off level of 0.01 exceeded by 16 subjects and for the 99th centile a cut-off level of 0.03 exceeded by 5 subjects. Subjects with IGT $(n=414)$ and diabetes $(n=18)$ representing abnormal OGTT $(n=434)$ had higher IA-2Ab index (mean rank 1138) compared with those with normal OGTT (mean rank 1060; $p=0.008$ ). Analysing men and women separately showed a difference between men, but not between women, with abnormal OGTT (mean rank 599) and men with normal OGTT (mean rank 539; $p=0.015)$.

Displacement study. Using rhGAD65 to displace the radioactive tracer showed that specific displacement was observed at the 30th percentile (Table 3). Note that above the 95th centile all GAD65 antibody binding was fully displaced showing that we detected specific GAD65Ab.

\section{Discussion}

In this population-based cross-sectional study the subjects were from a population dominated by people of Central-Swedish origin even if there is an admixture of people of Finnish and Saamish descent in the region [13]. The study is unique in its design since the study group is older than in previous studies and from unselected primary health care. Our primary finding was the relation between GAD65Ab and IA$2 \mathrm{Ab}$ and a pathological OGTT. This could signify an increased risk for developing Type I diabetes. The subjects diagnosed with IGT or diabetes after undergoing an OGTT had not reported any clinical symptoms of diabetes before the test and it is possible that they were in an early phase of developing hyper-
Table 3. Analysis of non-specific binding in the GAD65Ab radiobinding assay

\begin{tabular}{llll}
\hline $\begin{array}{l}\text { GAD65Ab } \\
\text { index }\end{array}$ & $\begin{array}{l}{ }^{35} \mathrm{~S}-\mathrm{GAD} 65 \\
\text { alone }\end{array}$ & $\begin{array}{l}{ }^{35} \mathrm{~S}-\mathrm{GAD} 65 \text { with } 150 \mathrm{nmol} / \mathrm{l} \\
\text { competitor }\end{array}$ \\
\cline { 2 - 4 } & & rhGAD65 & Proinsulin \\
\hline$\leq 95^{\text {th }}$ centile & $0.003(0.02)$ & $-0.0004(0.01)^{\mathrm{a}}$ & $0.003(0.01) \mathrm{NS}$ \\
$>95^{\text {th }}$ centile & $0.40(0.45)$ & $0.13(0.23)^{\mathrm{a}}$ & $0.39(0.47) \mathrm{NS}$ \\
\hline
\end{tabular}

Mean values of GAD65Ab index, standard deviation and significance between labelled GAD65 alone and competitors for each centile group are shown. ${ }^{\mathrm{a}}=p<0.001$

glycaemia. We therefore suggest that after a positive screening result for diabetes or IGT by OGTT, analysis of both GAD65Ab and IA-2Ab should be included in a second step to identify subjects at risk for Type I diabetes.

We are also able to present the prevalence of GA$\mathrm{D} 65 \mathrm{Ab}$ and $\mathrm{IA}-2 \mathrm{Ab}$ in an unselected population. The observation that increased BMI was related to concentrations of GAD65Ab was unsuspected. This was most prominent in older women but also in middle-aged men. A similar observation was made in the United Kingdom prospective diabetes study group [14] where it was found that only the presence of autoantibodies predicted an increased likelihood of insulin requirement in older adults. This in contrast to a recent report from Japan [15] in patients with diabetes and secondary failure to treatment with sulphonylurea, showing that subjects with BMI less than 26 had higher GAD65Ab concentrations than subjects with BMI more than 26. Our findings do not corroborate this report which is not surprising since we studied healthy subjects. Autoimmune markers have also been reported to be more prevalent in non-obese than in obese newly diagnosed adult Swedish diabetic patients [16]. Healthy subjects were not, however, investigated in these studies. There is no obvious explanation for the indication that obesity could possibly be correlated with increased GAD65Ab. In vitro experiments have shown that glucose increases islet expression of GAD65 $[17,18]$. In these reports it was speculated that high blood glucose concentrations possibly increase the functional activity of the beta cells which might stimulate the expression of the GAD65 autoantigen. It is also known that obesity is associated with insulin resistance and perhaps thereby there is a resulting increase in beta-cell activity. Therefore, GAD65Ab in obese subjects may mark subtle beta-cell dysfunction and insulin resistance in some of our subjects. It was not possible in this study to test this hypothesis since serum insulin was not measured.

Since some of our findings are made in subjects with low concentrations of GAD65Ab the accuracy of the method itself could be questionable. Our displacement study shows, however, that our GAD65Ab 
analysis is indeed specific. This was true for GAD65Ab concentrations down to about the 30th centile. This may seem surprising but similar low concentrations of antibodies in the general population are well known for, for example, thyroglobulin. Low levels of autoantibodies could therefore be a common phenomenon. Since the method for GAD65Ab IA$2 \mathrm{Ab}$ analysis is based on a final filtration step we also suspected that serum lipids or glucose concentrations might interfere with the antibody binding. This seems not to be the case since there was no relation between serum lipid or fasting plasma glucose concentrations and GAD65Ab or IA-2Ab indexes. Our results indicate that GAD65Ab do not differ in prevalence between younger and older subjects even if the GAD65Ab index, which reflects antibody levels tended to be higher among 60-year-old than 30-yearold subjects. These results imply that analysing GA$\mathrm{D} 65 \mathrm{Ab}$ IA-2Ab as a marker for Type I diabetes is of value at all ages. It is perhaps beneficial to patients with newly diagnosed diabetes mellitus to be tested for GAD65Ab to assist in the classification of the disease. Further GAD65Ab-positive diabetic patients would probably benefit from early insulin treatment [19].

In conclusion, in our study we present the prevalence of GAD65Ab and IA-2Ab in subjects aged either $30,40,50$ or 60 years. We show; firstly GAD65Ab and IA-2Ab are associated with impaired or diabetic glucose tolerance as detected by OGTT in an adult population, and secondly, high BMI is related to increased concentration of GAD65Ab irrespective of OGTT. This observation indicates a possible relation between islet cell autoimmunity and betacell function abnormalities associated with obesity causing insulin resistance.

Acknowledgements. This study was supported by grants from the Västerbotten County Council, the Elsa and Folke Sahlbergs Foundation, Umeå University, Swedish Council of Forestry and Agricultural Research (SJFR) and the National Institutes of Health (DK42654, DK 26190). The samples were analysed in the Immunoassay Core of the Diabetes Endocrinology Research Center of the University of Washington (DK17047). We gratefully acknowledge the help of $\AA$. Agren at the Department of Nutritional Research and Pathology, Umeå University for decoding the identities of the subjects and keeping the records in order.

\section{References}

1. World Health Organisation Study Group on Diabetes Mellitus (1994) Technical report series No. 844, WHO, Geneva

2. Landin Olsson M, Karlsson FA, Lernmark A, Sundkvist G (1992) Islet cell and thyrogastric antibodies in 633 consecutive 15- to 34-yr-old patients in the diabetes incidence study in Sweden. Diabetes 41: 1022-1027

3. Rabin DU, Pleasic SM, Palmer Crocker R, Shapiro JA (1992) Cloning and expression of IDDM-specific human autoantigens. Diabetes 41: 183-186
4. Verge CF, Gianani R, Kawasaki E (1996) Prediction of type I diabetes in first-degree relatives using a combination of insulin, GAD, and ICA512bdc/IA-2 autoantibodies. Diabetes 45: 926-933

5. Hagopian WA, Sanjeevi CB, Kockum I (1995) Glutamate decarboxylase-, insulin-, and islet cell-antibodies and HLA typing to detect diabetes in a general population-based study of Swedish children. J Clin Invest 95: 1505-1511

6. Hagopian WA, Karlsen AE, Gottsater A (1993) Quantitative assay using recombinant human islet glutamic acid decarboxylase (GAD65) shows that $64 \mathrm{~K}$ autoantibody positivity at onset predicts diabetes type. J Clin Invest 91: 368-374

7. Tuomi T, Groop LC, Zimmet PZ, Rowley MJ, Knowles W, Mackay IR (1993) Antibodies to glutamic acid decarboxylase reveal latent autoimmune diabetes mellitus in adults with a non-insulin-dependent onset of disease. Diabetes 42: $359-362$

8. Irvine WJ, Sawers JS, Feek CM, Prescott RJ, Duncan LJ (1979) The value of islet cell antibody in predicting secondary failure of oral hypoglycaemic agent therapy in Diabetes mellitus. J Clin Lab Immunol 2: 23-26

9. Niskanen LK, Tuomi T, Karjalainen J, Groop LC, Uusitupa MI (1995) GAD antibodies in NIDDM. Ten-year followup from the diagnosis. Diabetes Care 18: 1557-1565

10. Falorni A, Ortqvist E, Persson B, Lernmark A (1995) Radioimmunoassays for glutamic acid decarboxylase (GAD65) and GAD65 autoantibodies using 35S or 3H recombinant human ligands. J Immunol Methods 186: 89-99

11. Payton MA, Hawkes CJ, Christie MR (1995) Relationship of the 37,000-and 40,000-M(r) tryptic fragments of islet antigens in insulin-dependent diabetes to the protein tyrosine phosphatase-like molecule IA-2 (ICA512). J Clin Invest 96: 1506-1511

12. Grubin CF, Daniels T, Toivola B (1994) A novel radioligand binding assay to determine diagnostic accuracy of isoform-specific glutamic acid decarboxylase antibodies in childhood IDDM. Diabetologia 37: 344-350

13. Nylander PO (1992) Ethnic heterogeneity of the northSwedish population, [Medical dissertation] Department of Medical genetics, Umeå University, Umeå

14. Turner R, Stratton I, Horton V (1997) UKPDS 25: autoantibodies to islet-cell cytoplasm and glutamic acid decarboxylase for prediction of insulin requirement in type 2 diabetes. UK Prospective Diabetes Study Group. Lancet 350: 1288-1293

15. Fukui M, Nakano K, Shigeta H (1997) Antibodies to Glutamic Acid Decarboxylase in Japanese diabetic patients with secondary failure of oral hypoglycaemic therapy. Diabet Med 14: 148-151

16. Gottsäter A, Landin Olsson M, Lernmark A, Fernlund P, Sundkvist G, Hagopian WA (1995) Glutamate decarboxylase antibody levels predict rate of beta-cell decline in adult-onset diabetes. Diabetes Res Clin Pract 27: 133-140

17. Velloso LA, Bjork E, Ballagi AE (1994) Regulation of GAD expression in islets of Langerhans occurs both at the mRNA and protein level. Mol Cell Endocrinol 102: 31-37

18. Björk E, Kämpe O, Andersson A, Karlsson F (1992) Expression of the $64 \mathrm{kDa} /$ glutamic acid decarboxylase rat islet cell autoantigen is influenced by the rate of insulin secretion. Diabetologia 35: 490-493

19. Kobayashi T, Nakanishi K, Murase T, Kosaka K (1996) Small doses of subcutaneous insulin as a strategy for preventing slowly progressive beta-cell failure in islet cell antibody-positive patients with clinical features of NIDDM. Diabetes 45: 622-626 